\section{JTI}

JOURNAL OF

TRAUMA AND INJURY

\title{
An Unusual Complication of Colonic Perforation Following Percutaneous Nephrostomy in a Grade IV Blunt Renal Injury Patient
}

Received: March 25, 2019

Revised: May 11, 2019

Accepted: May 30, 2019

\section{Correspondence to}

Joan Gan Cheau Yan, M.B.B.S

General Surgery Department,

Hospital Sultanah Aminah,

Jalan Abu Bakar,

80100 Johor Bahru,

Johor Darul Takzim

Malaysia

Tel: +60-12-700-7995

Fax: +60-7-226-1028

E-mail:cyjoan2020@gmail.com

\author{
Joan Gan Cheau Yan, M.B.B.S. ${ }^{1,2}$, Tan Jih Huei, M.R.C.S. ${ }^{1,2}$, \\ Henry Tan Chor Lip, M.D. ${ }^{1,2}$, Yuzaidi Mohamad, M.Med Surg ${ }^{1}$, \\ Rizal Imran Alwi, M.Med Surg ${ }^{1}$ \\ ${ }^{1}$ General Surgery Department, Hospital Sultanah Aminah, Johor Bahru, Malaysia \\ ${ }^{2}$ Pusat Perubatan Universiti Kebangsaan Malaysia, Cheras, Malaysia
}

Percutaneous nephrostomy is relatively safe for temporary urinary diversion. However, colonic perforation due to percutaneous nephrostomy can happen with an incidence of $0.2 \%$ as reported in the English literatures. To our knowledge, this is the first case being reported as a complication following treatment for traumatic renal injury. This paper is to share our treatment approach which differs from the usual approach according to existing literatures. We report on a young man who sustained grade IV renal injury due to blunt trauma and was managed conservatively. The treatment of traumatic renal injury via urinary diversion was complicated with an iatrogenic colonic perforation. The management and subsequent treatment of this patient is discussed in this case report.

Keywords: Nephrostomy; Iatrogenic disease; Kidney; Urinary diversion; Wound, Nonpenetrating

\section{INTRODUCTION}

Colonic perforation after percutaneous nephrostomy is an uncommon complication but carries significant morbidity. It is often reported in non-traumatic patient with obstructive renal stone disease [1]. To our knowledge, there was no case report of percutaneous nephrostomy causing colonic perforation in patients with traumatic renal injury. This case reports on the uncommon complication that occurred in a young man with grade IV renal injury treated initially with percutaneous nephrostomy who (http://creativecommons.org/licenses/by-nc/4.0/) which permits unrestricted noncommercial use, distribution, and reproduction in any medium, provided the original work is properly cited. 
subsequently required nephrectomy and colonic repair.

\section{CASE REPORT}

A 31-year-old man presented with right sided abdominal pain following a high impact road traffic accident when he collided with a car while riding his motorcycle. On presentation, his vital parameters were within normal limits. Clinical examination revealed localized abdominal tenderness over the right flank. Focused Assessment Sonography in Trauma revealed the presence of free fluid at the Morrison's pouch. Contrasted computed tomography (CT) abdomen revealed a grade III liver injury, grade IV right renal injury with lower calyceal injury and urinary extravasation (Fig. 1). Non-operative management was taken for this patient as he was haemodynamically stable.

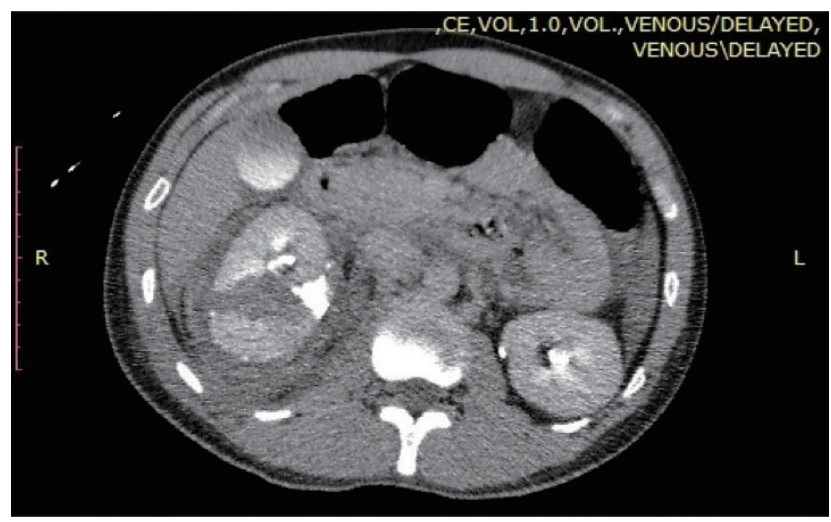

Fig. 1. Right renal injury with perinephric hematoma.

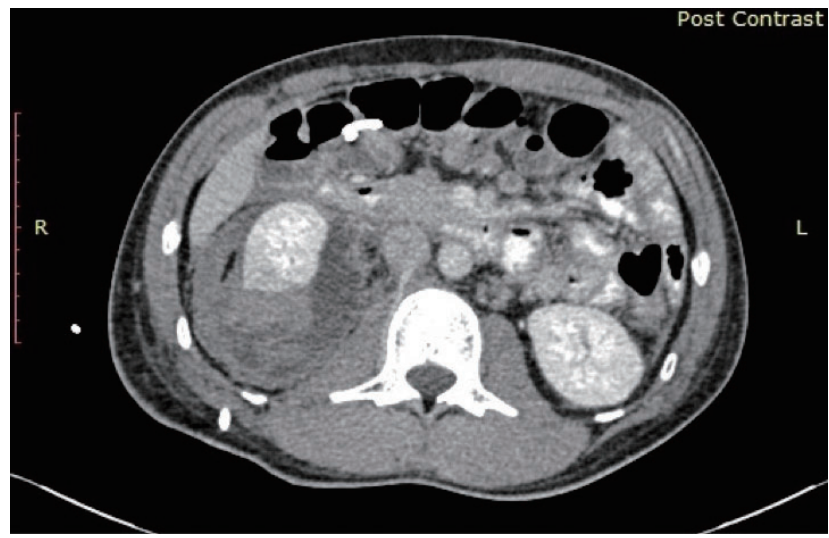

Fig. 2. Right perinephric hematoma expanded compared to day 1 scan.
However, due to worsening abdominal pain a repeat CT scan of the abdomen was performed which revealed an enlarging perinephric collection (Fig. 2). An attempt was made at retrograde ureteral stenting initially for urinary diversion as decided by the attending urologist in view of persistent urinary extravasation from the injured pelvicalyceal system. However, the attempt was unsuccessful and a percutaneous nephrostomy was requested. Due to technical difficulty, the cannulation of pelvic-calyceal system was unsuccessful and the tip of a pigtail catheter (sized $8 \mathrm{Fr}$ ) was placed within the perinephric collection instead for drainage of the resultant urinoma.

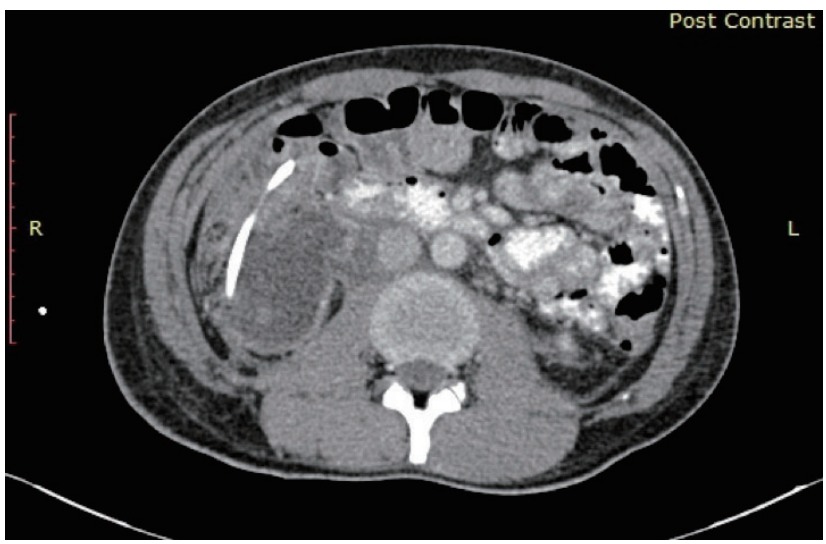

Fig. 3. Axial cut section of computed tomography abdomen showing the tip of pig tail catheter traversing into right colon.

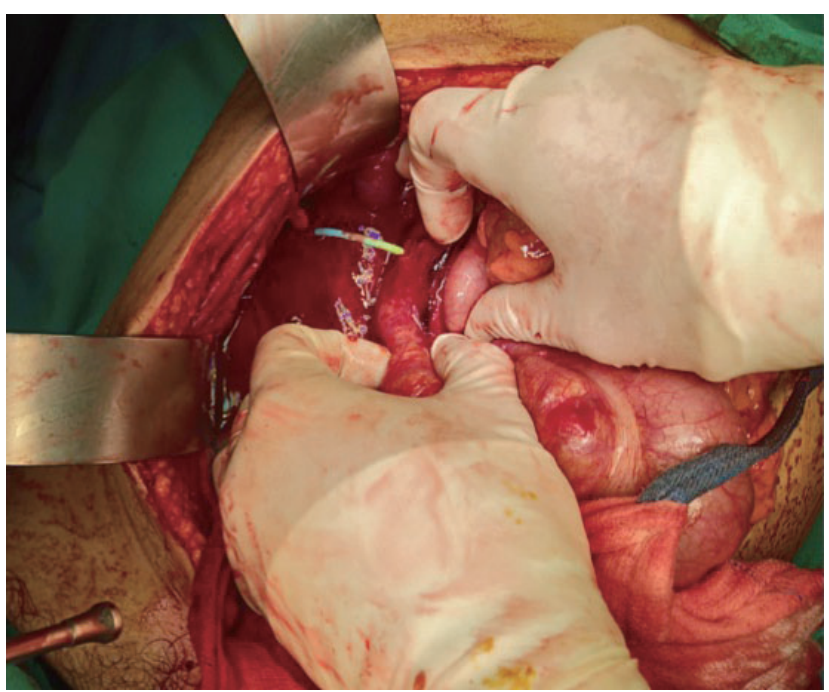

Fig. 4. Intraoperative picture shown the blue color pigtail catheter traversing from the right kidney towards the posterior surface of ascending colon. 
On day 2 post procedure, there was presence of fecal matter draining from the catheter and the patient became clinically septic as evident by temperature spike and raised total white count. A repeat CT scan showed colonic perforation with the pigtail catheter traversing the wall of the large bowel with its tip within the large bowel lumen and an expanding perinephric collection (Fig. 3). An urgent laparotomy was performed for large bowel repair and right nephrectomy. Intraoperative, the pigtail catheter is seen traversing through the perinephric space and puncturing into the ascending colon (Fig. 4). The catheter was removed and the perforation on the ascending colon was repaired primarily with absorbable sutures. The right retroperitoneal space (Zone II) was subsequently explored, and perinephric infected hematoma and urinoma was drained, debrided and washed following right nephrectomy. Post-operatively he was nursed in the general ward. Recovery was uneventful and he was discharged on post-operative day 7 .

\section{DISCUSSION}

Percutaneous nephrostomy (PCN) is a commonly practiced procedure especially in treating urological diseases such as urinary obstruction secondary to calculus, stricture or tumour. The procedure has been known to be safe and has a low complication rate. Cases of colonic perforation have been reported with an incidence of $0.2 \%$ [2]. Post procedural peritonitis is rare as most reported perforations were retroperitoneal. Conservative management is possible with total parenteral nutrition, bowel rest and antibiotic therapy. Surgery is indicated in patients who subsequently develop abscess or colocutaneous fistula.

With the advancement of minimally invasive techniques, it is recognized that grade IV renal trauma can be managed non-operatively. The aim in management is to maintain haemodynamic stability, relief of obstructed urinary flow and prevention of persistent urinary extravasation [3]. The review by European Association of Urology on renal trauma concluded urinary extravasation secondary to blunt renal trauma in a haemodynamic stable patient should be managed with endourologic or percutaneous techniques such as ureteral stent with or without percutaneous drainage of urinoma to allow healing to take place [4]. Prakash et al. [3] reported grade IV renal trauma can be successfully managed either by diversion with or without drainage with minimally invasive treatment method to salvage the injured kidney. In this current case, we attempted the both ureteral stenting and percutaneous drainage. However, the percutaneous technique had resulted in a colonic perforation. The authors could not find any literature in regards to percutaneous nephrostomy/drainage in traumatic renal injury leading to colonic perforation.

There is no evidence to show superiority of retrograde ureteral stent and percutaneous nephrostomy for urinary diversion. Both are acceptable treatment in such instances. The latest evidence suggests PCN may be a better option only in long-term urinary diversion as patients experience less urinary symptoms [5].

Nephrectomy is only indicated in traumatic renal injury with haemodynamic instability. Davis et al. [6] concluded that more than $50 \%$ of traumatic patients undergoing laparotomy for associated injury in blunt abdominal trauma did not require nephrectomy. Nephrectomy is indicated if intraoperatively there is expanding perinephric haematoma or pulsatile perinephric haematoma. In our patient, exploring the colonic injury required the mobilization of the ascending colon, a maneuver which would expose the retroperitoneal space. This would dislodge the clot on the injured kidney and expose the pelvic -calyceal system to the intraperitoneal space leading to urinary peritonitis. Finally, this is a grade IV renal trauma in which conservative management has failed. Hence the decision was made for right nephrectomy.

There have been reports on successful conservative management of retroperitoneal colonic perforation after percutaneous nephrolithotomy [7]. But those patients are usually kept nil per oral with total parenteral nutrition, initiation of broad-spectrum antibiotics, placement of Foley's catheter to relieve the pressure in urinary system, the nephrocolonic communication must be separated via different draining system. Other reports recommend a temporary diverting colostomy and patient is closely monitored for any signs of peritonitis or sepsis [7]. In contrary, we performed surgical exploration and primary repair of the retroperitoneal colonic perforation for our patient as 
he was showing clinical signs of peritonitis. Decision was made to repair the bowel perforation primarily due to the small size of perforation and minimal contamination. The early surgical intervention for our patient allowed him to return home earlier. There was no literature available for primary repair of retroperitoneal bowel perforation secondary to percutaneous nephrostomy.

Some risk factors have been reported in colonic injury following percutaneous nephrolithotomy for renal stone such as advanced age, markedly dilated pelvic-calyceal system, megacolon, anatomy variant such as retrorenal or posterolateral position of the colon [8]. In our case, the patient did not have any of the aforementioned risk factors. The colonic perforation in this case could possibly be due to misidentification of the anatomy due to distorted anatomy as a result of renal injury.

Management of persistent urinary extravasation in grade IV renal injury includes percutaneous or endoscopic diversion, drainage or surgery. No report or guideline can recommend one method over the other. However, a multi modal approach may be needed in cases where there is failure of resolution from one method or if complication develops. The case at hand demonstrates a multi modal approach as a result of complication arising from an attempt at urinary diversion in a non-dilated pelvic calyceal system which eventually required surgical rectification.

\section{REFERENCES}

1. Michel MS, Trojan L, Rassweiler JJ. Complications in percutaneous nephrolithotomy. Eur Urol 2007;51:899-906.

2. Dagli M, Ramchandani P. Percutaneous nephrostomy: technical aspects and indications. Semin Intervent Radiol 2011;28:424-37.

3. Prakash SV, Mohan CG, Reddy VB, Reddy VK, Kumar A, Reddy UM. Salvageability of kidney in grade IV renal trauma by minimally invasive treatment methods. J Emerg Trauma Shock 2015;8:16-20.

4. Serafetinides E, Kitrey ND, Djakovic N, Kuehhas FE, Lumen N, Sharma DM, et al. Review of the current management of upper urinary tract injuries by the EAU trauma guidelines panel. Eur Urol 2015;67:930-6.

5. Chang HC, Tang SH, Chuang FP, Wu ST, Sun GH, Yu DS, et al. Comparison between the use of percutaneous nephrostomy and internal ureteral stenting in the management of long-term ureteral obstructions. Urol Sci 2012;23:82-4.

6. Davis KA, Reed RL 2nd, Santaniello J, Abodeely A, Esposito TJ, Poulakidas SJ, et al. Predictors of the need for nephrectomy after renal trauma. J Trauma 2006;60:164-9.

7. Öztürk H. Treatment of colonic injury during percutaneous nephrolithotomy. Rev Urol 2015;17:194-201.

8. AslZare M, Darabi MR, Shakiba B, Gholami-Mahtaj L. Colonic perforation during percutaneous nephrolithotomy: an 18-year experience. Can Urol Assoc J 2014;8:E323-6. 